\title{
Epsilon co-function for planar mechanisms
}

Avdo Voloder, Elvedin Kljuno*

Mechanical Engineering Faculty, University of Sarajevo, Sarajevo, Bosnia and Herzegovina

\section{A R T I C L E IN F O}

\section{Article history:}

Received 13 March 2018

Received in revised form

20 May 2018

Accepted 22 June 2018

Keywords:

Angular acceleration

Angular velocity

Epsilon co-function

Bar mechanism

\begin{abstract}
A B S T R A C T
One of the most important and most challenging tasks of a mechanism analysis is the problem of mechanism kinematics. This paper shows the way to determine unknown angular accelerations of joint-bar mechanism components, by applying so-called (by authors) epsilon co-function. Using this method, the problem is reduced onto an analysis of relative angular accelerations of neighboring members within the mechanism and determination of a moment of all those vectors with respect to a point or an axis. The main contribution of this paper is that it shows the novel method how to calculate angular accelerations of mechanism members using analog form of equations that are similar to the moment balance equations in statics. Considering that the relative angular velocity vectors play role of forces in statics, this paper shows how to form a system of kinematic equations similar to moment equations in statics, which are sufficient to solve for all angular accelerations of a mechanism.
\end{abstract}

(C) 2018 The Authors. Published by IASE. This is an open access article under the CC BY-NC-ND license (http://creativecommons.org/licenses/by-nc-nd/4.0/).

\section{Introduction}

Several methods exist in the theory of planar mechanisms to determine particular kinematic quantities (such as angular velocity and angular acceleration) of the mechanism members or particular points of the mechanism, such as: using a particle kinematics, using planar kinematics equations, using the method of complex numbers and using the method of mechanism reduction. Each of these methods has some advantages with respect to others, depending on the type of the problem that should be solved.

Particularly, there are methods that use relative motion of neighboring members to determine unknown angular velocities of a hinge-lever mechanism (Ilic, 1966; Hufnagl, 1974; Voloder, 2005).

\section{Important facts about the $\vec{\omega}$ co-function}

Let us assume that a planar mechanical system is consisted of $n$ rigid bodies (members) that are mutually interconnected. We will analyze relative angular velocities and relative angular accelerations of all pairs out of $n$ observed members in a cyclic order (Fig. 1).

\footnotetext{
* Corresponding Author

Email Address: kljuno@mef.unsa.ba (E. Kljuno)

https://doi.org/10.21833/ijaas.2018.09.002

2313-626X/C) 2018 The Authors. Published by IASE.

This is an open access article under the CC BY-NC-ND license

(http://creativecommons.org/licenses/by-nc-nd/4.0/)
}

If we observe the set of relative angular velocities of sequential members, given by the following equations

$$
\begin{aligned}
& \vec{\omega}_{2,1}=\vec{\omega}_{2}-\vec{\omega}_{1}, \\
& \vec{\omega}_{3,2}=\vec{\omega}_{3}-\vec{\omega}_{2}, \\
& \vec{\omega}_{n, n-1}=\vec{\omega}_{n}-\vec{\omega}_{n-1}, \\
& \vec{\omega}_{1, n}=\vec{\omega}_{1}-\vec{\omega}_{n},
\end{aligned}
$$

such a set of relative angular velocity vectors is called the $\vec{\omega}$ co-function with respect to $n$ members (Hufnagl, 1974).

If we add all relative angular velocities, according to (1), we obtain the following vector equation

$\vec{\omega}_{2,1}+\vec{\omega}_{3,2}+\cdots+\vec{\omega}_{n, n-1}+\vec{\omega}_{1, n}=\overrightarrow{0}$

which yields

$\sum_{1}^{n} \vec{\omega}_{i+1, i}=\overrightarrow{0}$,

where the index $i=n+1$ denotes the same vector as for $i=1$, due to the periodicity of the index.

The main vector of the moment of $\vec{\omega}$ co-function for an arbitrary point as a pole is equal zero

$\sum_{1}^{n} \vec{M}_{O}^{\vec{\omega}_{i+1, i}}=\overrightarrow{0}$

Equations (1) and (2) show that the vectors of the $\vec{\omega}$ co-function have same properties as an arbitrary balanced system of forces. In this way, the same 

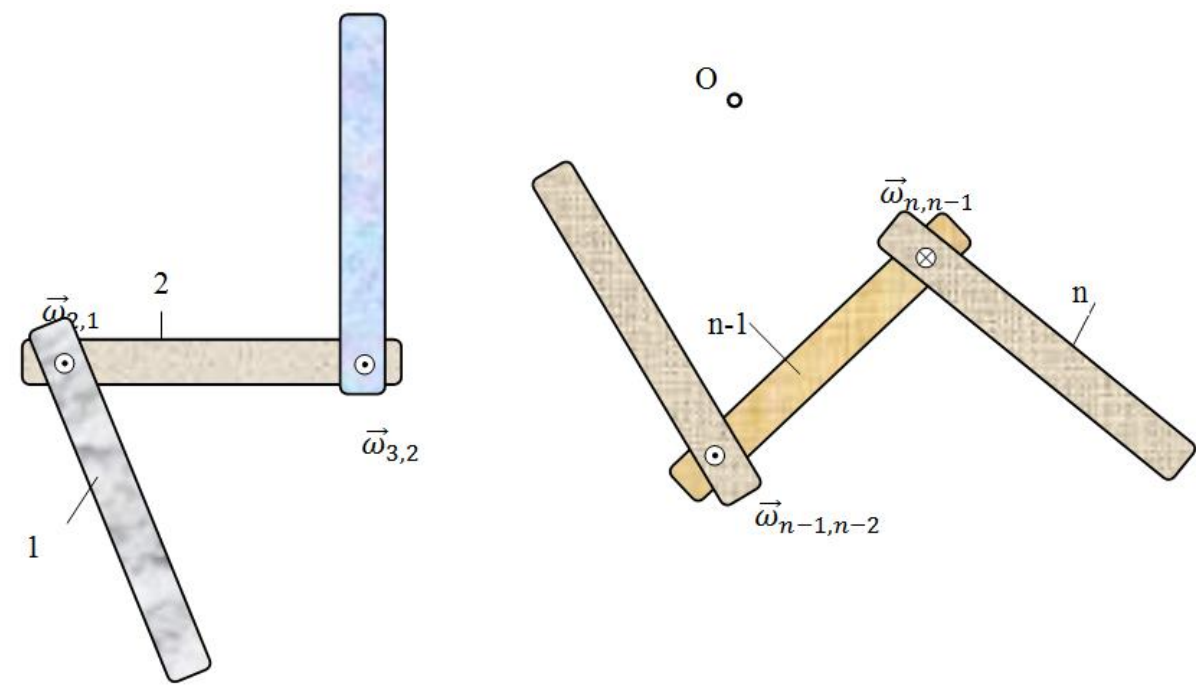

Fig. 1: Vectors of the $\vec{\omega}$ - co-function

\section{3. $\vec{\epsilon}$ co-function}

\subsection{Definition of $\vec{\epsilon}$ co-function}

Let us observe a set of relative angular accelerations of sequential members (Fig. 2), given by the following equations

$\vec{\epsilon}_{2,1}=\vec{\epsilon}_{2}-\vec{\epsilon}_{1}$

$$
\begin{aligned}
& \vec{\epsilon}_{3,2}=\vec{\epsilon}_{3}-\vec{\epsilon}_{2}, \\
& \vec{\epsilon}_{n, n-1}=\vec{\epsilon}_{n}-\vec{\epsilon}_{n-1}, \\
& \vec{\epsilon}_{1, n}=\vec{\epsilon}_{1}-\vec{\epsilon}_{n} .
\end{aligned}
$$

Such a set of relative angular acceleration vectors we will call $\vec{\epsilon}$ co-function, similarly to the previous $\vec{\omega}$ co-function. Now, we will prove some properties of the $\vec{\epsilon}$ co-function.
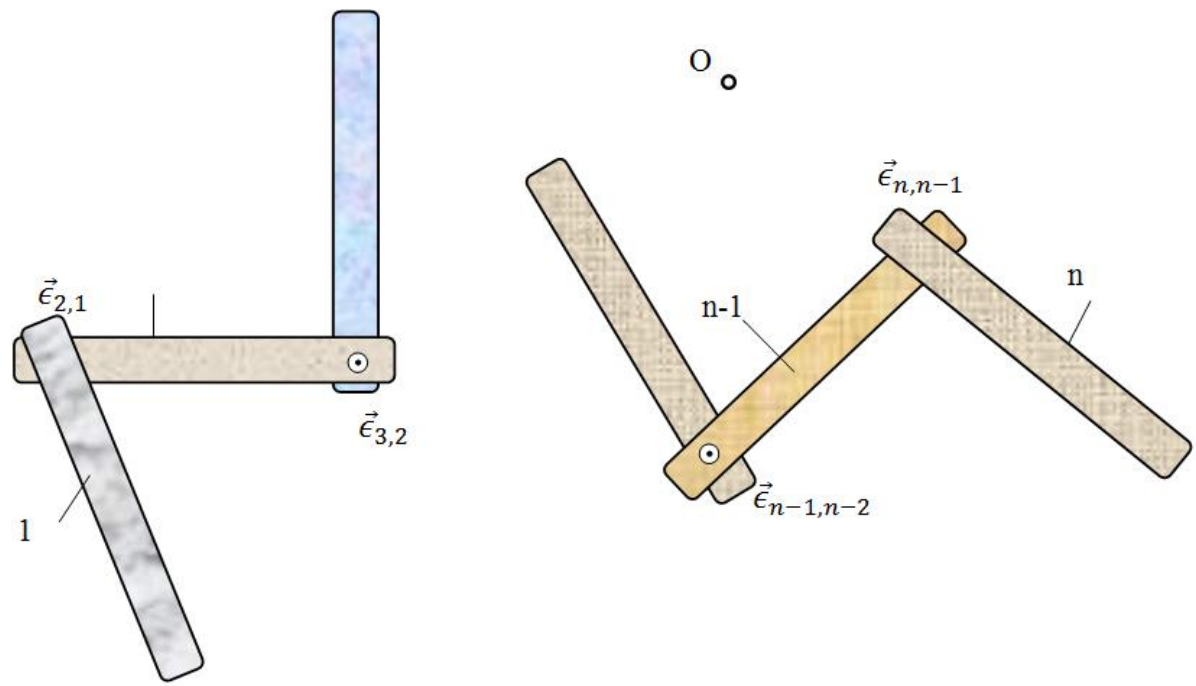

Fig. 2: Vectors of the $\vec{\epsilon}$ - co-function

By summing up Eq. (2), we obtain the following vector equation

$\vec{\epsilon}_{2,1}+\vec{\epsilon}_{3,2}+\cdots+\vec{\epsilon}_{n, n-1}+\vec{\epsilon}_{1, n}=\overrightarrow{0}$

which yields

$\sum_{1}^{n} \vec{\epsilon}_{i+1, i}=\overrightarrow{0}$

If the first member is denoted as 0 , then the sum in (4) starts with 0, e.g. if the ground member is assigned with 0 .

\subsection{Moment of $\vec{\epsilon}$ co-function for an arbitrary pole}

Equation (4) shows that the principal vector (the vector sum) of the angular accelerations in $\vec{\epsilon}$ cofunction is equal zero, hence this property is similar to the first property of the $\vec{\omega}$ co-function.

However, when the moment of the $\vec{\epsilon}$ co-function vector is considered with respect to an arbitrary point 0 , then significant difference can be noticed when compared with the corresponding property of the $\vec{\omega}$ co-function. The following analysis will show this difference. 
Let us observe members: $(i-1),(i),(i+1)$ of a selected set of members (Fig. 3). Assuming that the point $P_{i, i-1}$ belongs simultaneously to members ( $i-$ 1 ) and $(i)$, then it is on the direction of relative angular acceleration $\vec{\epsilon}_{i, i-1}$. Similarly, let us assume the same for the point $P_{i+1, i}$ and members $(i),(i+$ 1).

Then, relative acceleration of points $P_{i+1, i}$ and $P_{i, i-1}$ is

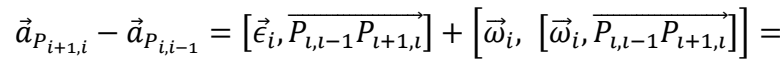
$\left[\overrightarrow{P_{l+1, l} P_{l, l-1},}, \vec{\epsilon}_{i}\right]+\left[\vec{\omega}_{i},\left[\vec{\omega}_{i}, \overrightarrow{P_{l, l-1} P_{l+1, l}}\right]\right]$ which is

$$
\begin{aligned}
& \vec{a}_{P_{2,1}}-\vec{a}_{P_{1, n}}=\left[\vec{\epsilon}_{1}, \overrightarrow{P_{1, n} P_{2,1}}\right]+\left[\vec{\omega}_{1},\left[\vec{\omega}_{1}, \overrightarrow{P_{1, n} P_{2,1}}\right]\right]= \\
& {\left[\overrightarrow{P_{2,1} P_{1, n}}, \vec{\epsilon}_{1}\right]+\left[\vec{\omega}_{1},\left[\vec{\omega}_{1}, \overrightarrow{P_{1, n} P_{2,1}}\right]\right] \text {, }} \\
& \vec{a}_{P_{3,2}}-\vec{a}_{P_{2,1}}=\left[\vec{\epsilon}_{2}, \overrightarrow{P_{2,1} P_{3,2}}\right]+\left[\vec{\omega}_{2},\left[\vec{\omega}_{2}, \overrightarrow{P_{2,1} P_{3,2}}\right]\right]= \\
& {\left[\overrightarrow{P_{3,2} P_{2,1}}, \vec{\epsilon}_{2}\right]+\left[\vec{\omega}_{2},\left[\vec{\omega}_{2}, \overrightarrow{P_{2,1} P_{3,2}}\right]\right] \text {, }} \\
& \vec{a}_{P_{n, n-1}}-\vec{a}_{P_{n-1, n-2}}=\left[\vec{\epsilon}_{n-1}, \overrightarrow{P_{n-1, n-2} P_{n, n-1}}\right]+\left[\vec{\omega}_{n-1},\right. \\
& \left.\left[\vec{\omega}_{n-1}, \overrightarrow{P_{n-1, n-2} P_{n, n-1}}\right]\right]=\left[\overrightarrow{P_{n, n-1} P_{n-1, n-2}}, \vec{\epsilon}_{n-1}\right]+ \\
& +\left[\vec{\omega}_{n-1},\left[\vec{\omega}_{n-1}, \overrightarrow{P_{n-1, n-2} P_{n, n-1}}\right]\right], \\
& \vec{a}_{P_{1, n}}-\vec{a}_{P_{n, n-1}}=\left[\vec{\epsilon}_{n}, \overrightarrow{P_{n, n-1} P_{1, n}}\right]+\left[\vec{\omega}_{n},\left[\vec{\omega}_{n}, \overrightarrow{P_{n, n-1} P_{1, n}}\right]\right]= \\
& {\left[\overrightarrow{P_{1, n} P_{n, n-1}}, \vec{\epsilon}_{n}\right]+\left[\vec{\omega}_{n},\left[\vec{\omega}_{n}, \overrightarrow{P_{n, n-1} P_{1, n}}\right]\right] \text {. }}
\end{aligned}
$$

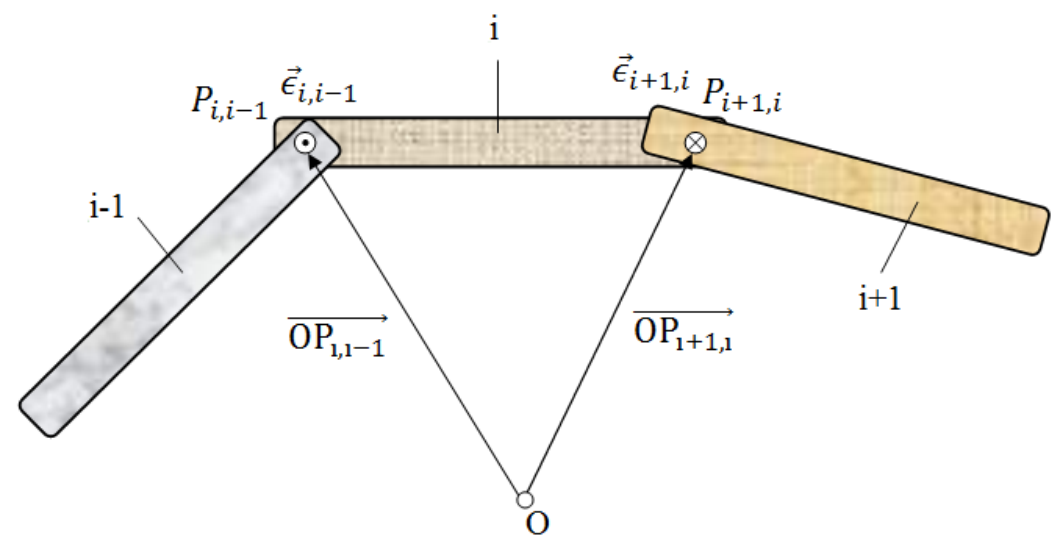

Fig. 3: With the proof of the principal moment of the $\vec{\epsilon}$ co-function

Due to the following

$\vec{a}_{P_{2,1}}-\vec{a}_{P_{1, n}}+\vec{a}_{P_{3,2}}-\vec{a}_{P_{2,1}}+\cdots+\vec{a}_{P_{n, n-1}}-\vec{a}_{P_{n-1, n-2}}+$

$\vec{a}_{P_{1, n}}-\vec{a}_{P_{n, n-1}}=\overrightarrow{0}$,

and using (5), we obtain

$\left[\overrightarrow{P_{2,1} P_{1, n}}, \vec{\epsilon}_{1}\right]+\left[\vec{\omega}_{1},\left[\vec{\omega}_{1}, \overrightarrow{P_{1, n} P_{2,1}}\right]\right]+\left[\overrightarrow{P_{3,2} P_{2,1}}, \vec{\epsilon}_{2}\right]+$ $\left[\vec{\omega}_{2},\left[\vec{\omega}_{2}, \overrightarrow{P_{2,1} P_{3,2}}\right]\right]+\cdots+\left[\overrightarrow{P_{n, n-1} P_{n-1, n-2},} \vec{\epsilon}_{n-1}\right]+$ $+\left[\vec{\omega}_{n-1},\left[\vec{\omega}_{n-1}, \overrightarrow{P_{n-1, n-2} P_{n, n-1}}\right]\right]+$

$\left[\overrightarrow{P_{1, n} P_{n, n-1}}, \vec{\epsilon}_{n}\right]+\left[\vec{\omega}_{n},\left[\vec{\omega}_{n}, \overrightarrow{P_{n, n-1} P_{1, n}}\right]\right]=\overrightarrow{0}$.

If we choose an arbitrary point as a pole 0 , then

$\overrightarrow{\mathrm{P}_{(1+1,1)} \mathrm{P}_{1,1-1}}=\overrightarrow{\mathrm{OP}_{1,1-1}}-\overrightarrow{\mathrm{OP}_{(1+1,1)}}$.

Using (7) and (8), we obtain

$\left[\left(\overrightarrow{\mathrm{OP}_{1, \mathrm{n}}}-\overrightarrow{\mathrm{OP}_{2,1}}\right), \vec{\epsilon}_{1}\right]+\left[\vec{\omega}_{1},\left[\vec{\omega}_{1}, \overrightarrow{\mathrm{P}_{1, \mathrm{n}} \mathrm{P}_{2,1}}\right]\right]+\left[\overrightarrow{\mathrm{OP}_{2,1}}-\right.$ $\left.\left.\overrightarrow{\mathrm{OP}_{3,2}}\right), \vec{\epsilon}_{2}\right]+\left[\vec{\omega}_{2},\left[\vec{\omega}_{2}, \overrightarrow{\mathrm{P}_{2,1} \mathrm{P}_{3,2}}\right]\right]+\cdots$

$+\left[\left(\overrightarrow{\mathrm{OP}_{\mathrm{n}-1, \mathrm{n}-2}}-\overrightarrow{\mathrm{OP}_{\mathrm{n}, \mathrm{n}-1}}\right), \vec{\epsilon}_{n-1}\right]+$

$\left[\vec{\omega}_{n-1},\left[\vec{\omega}_{n-1}, \overrightarrow{\mathrm{P}_{\mathrm{n}-1, \mathrm{n}-2} \mathrm{P}_{\mathrm{n}, \mathrm{n}-1}}\right]\right]+\left[\left(\overrightarrow{\mathrm{OP}_{\mathrm{n}, \mathrm{n}-1}}-\overrightarrow{\mathrm{OP}_{1, \mathrm{n}}}\right), \vec{\epsilon}_{n}\right]+$

$+\left[\vec{\omega}_{n},\left[\vec{\omega}_{n}, \overrightarrow{\mathrm{P}_{\mathrm{n}, \mathrm{n}-1} \mathrm{P}_{1, \mathrm{n}}}\right]\right]=\overrightarrow{0}$,

$\left[\overrightarrow{\mathrm{OP}_{2,1}},\left(\vec{\epsilon}_{2}-\vec{\epsilon}_{1}\right)\right]+\left[\overrightarrow{\mathrm{OP}_{3,2}},\left(\vec{\epsilon}_{3}-\vec{\epsilon}_{2}\right)\right]+\cdots+\left[\overrightarrow{\mathrm{OP}_{\mathrm{n}, \mathrm{n}-1}},\left(\vec{\epsilon}_{n}-\right.\right.$ $\left.\left.\vec{\epsilon}_{n-1}\right)\right]+\left[\overrightarrow{\mathrm{OP}_{1, \mathrm{n}}},\left(\vec{\epsilon}_{1}-\vec{\epsilon}_{n}\right)\right]+$

$+\left[\vec{\omega}_{1},\left[\vec{\omega}_{1}, \overrightarrow{\mathrm{P}_{1, \mathrm{n}} \mathrm{P}_{2,1}}\right]\right]+\left[\vec{\omega}_{2},\left[\vec{\omega}_{2}, \overrightarrow{\mathrm{P}_{2,1} \mathrm{P}_{3,2}}\right]\right]+\cdots+$

$\left[\vec{\omega}_{n-1},\left[\vec{\omega}_{n-1}, \overrightarrow{\mathrm{P}_{\mathrm{n}-1, \mathrm{n}-2} \mathrm{P}_{\mathrm{n}, \mathrm{n}-1}}\right]\right]+\left[\vec{\omega}_{n},\left[\vec{\omega}_{n}, \overrightarrow{\mathrm{P}_{\mathrm{n}, \mathrm{n}-1} \mathrm{P}_{1, \mathrm{n}}}\right]\right]=\overrightarrow{0}$,
$\left[\overrightarrow{\mathrm{OP}_{2,1}}, \vec{\epsilon}_{2,1}\right]+\left[\overrightarrow{\mathrm{OP}_{3,2},}, \vec{\epsilon}_{3,2}\right]+\cdots+\left[\overrightarrow{\mathrm{OP}_{\mathrm{n}, \mathrm{n}-1}}, \vec{\epsilon}_{n, n-1}\right]+$

$\left[\overrightarrow{\mathrm{OP}_{1, n}}, \vec{\epsilon}_{1, n}\right]+\left[\vec{\omega}_{1},\left[\vec{\omega}_{1}, \overrightarrow{\mathrm{P}_{1, \mathrm{n}} \mathrm{P}_{2,1}}\right]\right]+$

$+\left[\vec{\omega}_{2},\left[\vec{\omega}_{2}, \overrightarrow{\mathrm{P}_{2,1} \mathrm{P}_{3,2}}\right]\right]+\cdots+\left[\vec{\omega}_{n-1},\left[\vec{\omega}_{n-1}, \overrightarrow{\mathrm{P}_{\mathrm{n}-1, \mathrm{n}-2} \mathrm{P}_{\mathrm{n}, \mathrm{n}-1}}\right]\right]+$

$\left[\vec{\omega}_{n},\left[\vec{\omega}_{n}, \overrightarrow{\mathrm{P}_{\mathrm{n}, \mathrm{n}-1} \mathrm{P}_{1, \mathrm{n}}}\right]\right]=\overrightarrow{0}$

Concise form of (11) is the following

$\sum_{1}^{n}\left[\overrightarrow{\mathrm{OP}_{1+1,1}}, \vec{\epsilon}_{i+1, i}\right]+\left[\vec{\omega}_{i},\left[\vec{\omega}_{i}, \overrightarrow{\mathrm{P}_{1,1-1} \mathrm{P}_{1+1,1}}\right]\right]=\overrightarrow{0}$,

and considering that:

a) the first term under the sum represents the moment of the relative angular acceleration vector with respect to the arbitrary point 0 , and

b) the second term represents the normal relative acceleration of the point $P_{i+1, i}$ with respect to the previous point in the sequence $P_{i, i-1}$, then (12) can be written in the following form

$\sum_{1}^{n} \vec{M}_{\mathrm{O}}^{\vec{\epsilon}_{i+1, i}}+\sum_{1}^{n} \underset{\mathrm{a}_{\mathrm{i}+1, i} \mathrm{P}_{\mathrm{i}, i-1}}{a x p}=\overrightarrow{0}$.

Equation (13) shows that the vector addition of the principal moment for an arbitrary pole 0 and all relative normal accelerations between sequential connection points of the mechanism.

\section{Example}

Bar 1 of the mechanism shown in Fig. 4 has a constant angular velocity: $\omega_{1}=20 \frac{\mathrm{rad}}{\mathrm{s}}$, and the bar 4 
has a constant angular velocity: $\omega_{4}=40 \frac{\mathrm{rad}}{\mathrm{s}}$. Using the described method of $\vec{\epsilon}$ co-function, angular accelerations of members 2 and 3 need to be determined. The following is given: $\overline{\mathrm{AB}}=\overline{\mathrm{DE}}=\overline{\mathrm{E} J}=$ $\overline{\mathrm{EH}}=L$, at the given instant the angles are $\angle \mathrm{BAD}=$ $60^{\circ}$ and $\angle \mathrm{ABD}=90^{\circ}$.

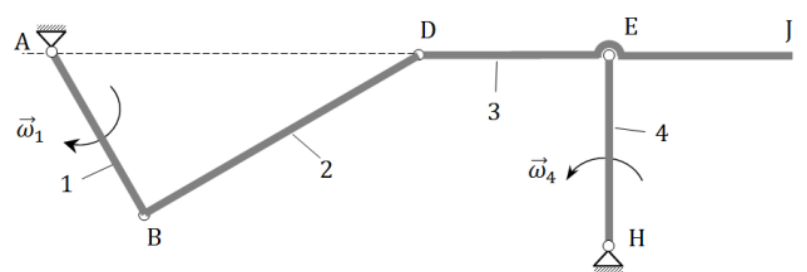

Fig. 4: Example: mechanism configuration

Solution can be given using the classic approach and the described method using the $\vec{\epsilon}$ co-function.

\subsection{Solution using classic method}

Using kinematics theory of planar motion, we can establish vector equations for velocities of mechanism connection points, as shown in Fig. 5.

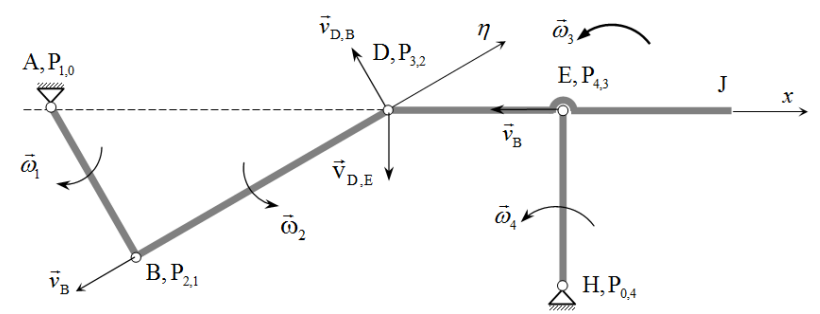

Fig. 5: Example: classic solution method - velocities

The velocity of point $\mathrm{D}$ can be expressed using the velocity of point $\mathrm{B}$ or $\mathrm{E}$ as

$\vec{v}_{D}=\vec{v}_{B}+\vec{v}_{D, B}=\vec{v}_{E}+\vec{v}_{D, E}$

where the shown velocity magnitudes in $\left[\frac{\mathrm{m}}{\mathrm{s}}\right],(L[\mathrm{~m}])$ are:

$v_{B}=L \omega_{1}=20 L, \quad v_{E}=L \omega_{1}=40 L, \quad v_{D, B}=\overline{\mathrm{DB}} \omega_{2}=$ $L \sqrt{3} \omega_{2}, v_{D, E}=\overline{\mathrm{DE}} \omega_{3}=L \omega_{3}$.

By projecting of (14) onto $x$ axis, we obtain

$-v_{B} \cos 30^{\circ}-v_{D, B} \sin 30^{\circ}=-v_{E}$,

$20 L \frac{\sqrt{3}}{2}+L \sqrt{3} \omega_{2} \frac{1}{2}=40 L, \omega_{2}=26.188 \frac{\mathrm{rad}}{\mathrm{s}}$.

Similarly, by projecting of (14) onto $\eta$ axis, we obtain

$-v_{B}=-v_{E} \cos 60^{\circ}-v_{D, E} \cos 60^{\circ}$,

$20 L=40 L \frac{\sqrt{3}}{2}+L \omega_{3} \frac{1}{2}, \omega_{3}=-29.282 \frac{\mathrm{rad}}{\mathrm{s}}$.

Angular accelerations can be obtained similarly, by using the vector loop equation, i.e., by expressing accelerations using accelerations of other points in the kinematic chain over connection points of the mechanism, as shown in Fig. 6.

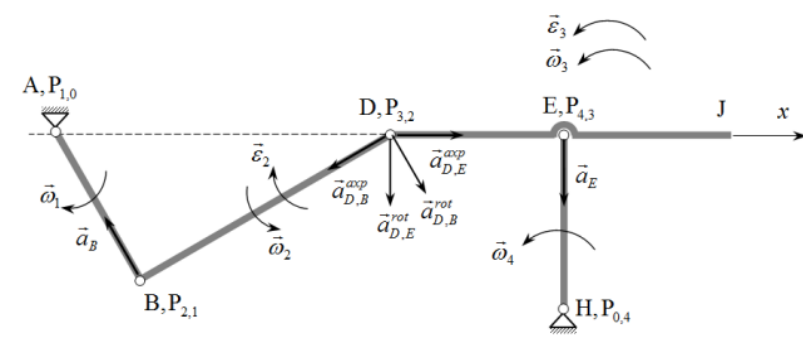

Fig. 6: Example: classic solution method - accelerations

The acceleration of the same connection point, e.g. the joint D, can be obtained using two different chains of points. In this way, we obtain

$\vec{a}_{D}=\vec{a}_{B}+\vec{a}_{D, B}^{a x p}+\vec{a}_{D, B}^{r o t}=\vec{a}_{E}+\vec{a}_{D, E}^{a x p}+\vec{a}_{D, E}^{r o t}$,

where the angular velocity magnitudes are determined earlier. The relative components are:

$a_{D, B}^{a x p}=\overline{D B} \omega_{2}^{2}=L \sqrt{3} 26.188^{2}=1187.860 L, \quad a_{D, E}^{a x p}=$

$\overline{D E} \omega_{3}^{2}=L 29.282^{2}=857.435 \mathrm{~L}$.

$a_{D, B}^{r o t}=\overline{D B} \epsilon_{2}=L \sqrt{3} \epsilon_{2}, a_{D, E}^{r o t}=\overline{D E} \epsilon_{3}=L \epsilon_{3}$.

Accelerations of the connection points B and E are equal to normal accelerations, with magnitudes (in $\left[\frac{\mathrm{m}}{\mathrm{s}^{2}}\right]$, where $L$ is in $[\mathrm{m}]$ ):

$a_{B}=\overline{A B} \omega_{1}^{2}=400 L, a_{E}=\overline{H E} \omega_{4}^{2}=1600 L$.

By projecting (18) onto $\mathrm{x}$ axis, we obtain

$-a_{B} \cos 60^{\circ}-a_{D, B}^{a x p} \sin 60^{\circ}+a_{D, B}^{r o t} \cos 60^{\circ}=a_{D, E}^{a x p}$,

$-400 L \frac{1}{2}-1187.860 L \frac{\sqrt{3}}{2}+L \sqrt{3} \epsilon_{2} \frac{1}{2}=857.435 L$,

$\epsilon_{2}=2408.880 \frac{\mathrm{rad}}{\mathrm{s}^{2}}$.

By projecting (18) onto $\eta$ axis, we obtain

$-a_{D, B}^{a x p}=-a_{E} \cos 60^{\circ}+a_{D, E}^{a x p} \cos 30^{\circ}-a_{D, E}^{r o t} \cos 60^{\circ}$,

$-1187.860 L=-1600 L \frac{1}{2}+857.435 L \frac{\sqrt{3}}{2}-L \epsilon_{3} \frac{1}{2}$,

$\epsilon_{3}=2260.840 \frac{\mathrm{rad}}{\mathrm{s}^{2}}$.

\subsection{Solution by applying the described method of $\vec{\omega}$ and $\vec{\epsilon}$ co-functions}

\subsubsection{Angular velocity via $\vec{\omega}$ - cofunction}

By projecting (2) onto $x$ axis (Fig. 7), we obtain

$M_{x}^{\vec{\omega}_{1,0}}+M_{x}^{\vec{\omega}_{2,1}}+M_{x}^{\vec{\omega}_{3,2}}+M_{x}^{\vec{\omega}_{4,3}}+M_{x}^{\vec{\omega}_{0,4}}=0$,

where the index starts at 0 , for the ground "member" and ends at 4.

Since the position vectors of the connection points $P_{1,0}, P_{3,2}, P_{4,3}$ are at $x$ axis (for $0 \equiv \mathrm{A}$ ), and vectors $\vec{\omega}_{1,0}, \vec{\omega}_{3,2}$, and $\vec{\omega}_{4,3}$ intersect $x$ axis, then there is no moment of those angular velocity vectors for $x$ axis, i.e,.

$M_{x}^{\vec{\omega}_{1,0}}=M_{x}^{\vec{\omega}_{3,2}}=M_{x}^{\vec{\omega}_{4,3}}=0$, 
and (27) becomes reduced to

$M_{x}^{\vec{\omega}_{2,1}}+M_{x}^{\vec{\omega}_{0,4}}=0$

Equation (29) becomes

$-\omega_{2,1} L \frac{\sqrt{3}}{2}+\omega_{0,4} L=0$.

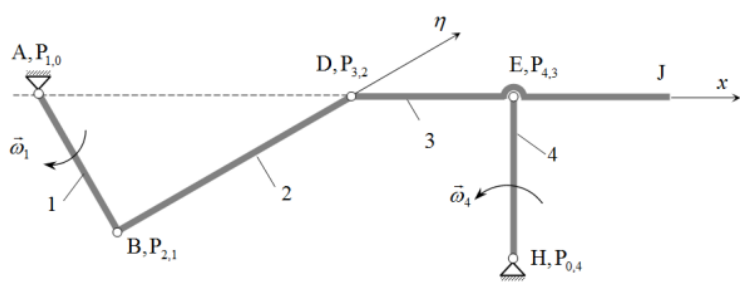

Fig. 7: Example: solution method using co-functions velocities

Considering that $\omega_{2,1}=\omega_{2}-\omega_{1}, \omega_{0,4}=\omega_{0}-\omega_{4}$, $\omega_{0}=0$, then (30) becomes

$\omega_{2}=\omega_{1}-\frac{2 \sqrt{3}}{3} \omega_{4}$

$\omega_{2}=-26.188 \frac{\mathrm{rad}}{\mathrm{s}}$.

Similarly, by projecting (2) onto $\eta$ axis, we obtain

$M_{\eta}^{\vec{\omega}_{1,0}}+M_{\eta}^{\vec{\omega}_{2,1}}+M_{\eta}^{\vec{\omega}_{3,2}}+M_{\eta}^{\vec{\omega}_{4,3}}+M_{\eta}^{\vec{\omega}_{0,4}}=0$.

Since $\omega_{0}=0$, then $\omega_{1,0}=\omega_{1}-\omega_{0}=\omega_{1}$, and (33) becomes

$M_{\eta}^{\vec{\omega}_{1}}+M_{\eta}^{\vec{\omega}_{2,1}}+M_{\eta}^{\vec{\omega}_{3,2}}+M_{\eta}^{\vec{\omega}_{4,3}}-M_{\eta}^{\vec{\omega}_{4}}=0$

Further, the points $P_{2,1}$ and $P_{3,2}$ are on the axis $\eta$, then

$M_{\eta}^{\vec{\omega}_{2,1}}=M_{\eta}^{\vec{\omega}_{3,2}}=0$

Using (35) and (34), we obtain

$M_{\eta}^{\vec{\omega}_{1}}+M_{\eta}^{\vec{\omega}_{4,3}}-M_{\eta}^{\vec{\omega}_{4}}=0$,

$-\omega_{1} L-\left(\omega_{4}-\omega_{3}\right) L \frac{1}{2}+\omega_{4}\left(L \frac{1}{2}+L \frac{\sqrt{3}}{2}\right)=0$,

$\omega_{3}=-29.282 \frac{\mathrm{rad}}{\mathrm{s}}$.

\subsubsection{Angular acceleration via $\vec{\epsilon}$ co-function}

By projecting (13) onto $x$ axis (Fig. 8), the following is obtained

$M_{x}^{\vec{\epsilon}_{1,0}}+M_{x}^{\vec{\epsilon}_{2,1}}+M_{x}^{\vec{\epsilon}_{3,2}}+M_{x}^{\vec{\epsilon}_{4,3}}+M_{x}^{\vec{\epsilon}_{0,4}}+\left[\vec{\omega}_{1}\right.$,

$\left.\left[\vec{\omega}_{1}, \overrightarrow{P_{1,0} P_{2,1}}\right]\right]_{x}+\left[\vec{\omega}_{2},\left[\vec{\omega}_{2}, \overrightarrow{P_{2,1} P_{3,2}}\right]\right]_{x}+\left[\vec{\omega}_{3}\right.$,

$\left.\left[\vec{\omega}_{3}, \overrightarrow{P_{3,2} P_{4,3}}\right]\right]_{x}++\left[\vec{\omega}_{4},\left[\vec{\omega}_{4}, \overrightarrow{P_{4,3} P_{0,4}}\right]\right]_{x}=0$,

where the terms are:

$M_{x}^{\vec{\epsilon}_{2,1}}=\left(\epsilon_{2}-\epsilon_{1}\right) L \sin 60^{0}=-\epsilon_{2} L \frac{\sqrt{3}}{2}, M_{x}^{\vec{\epsilon}_{3,2}}=0, M_{x}^{\vec{\epsilon}_{4,3}}=0$, $M_{x}^{\vec{\epsilon}_{0,4}}=0, M_{x}^{\vec{\epsilon}_{1,0}}=0$, $\left[\vec{\omega}_{1},\left[\vec{\omega}_{1}, \overrightarrow{P_{1,0} P_{2,1}}\right]\right]_{x}=-\omega_{1}^{2} \overline{A B} \cos 60^{\circ}=-200 \mathrm{~L}$,

$\left[\vec{\omega}_{2},\left[\vec{\omega}_{2}, \overrightarrow{P_{2,1} P_{3,2}}\right]\right]_{x}=-\omega_{2}^{2} \overline{B D} \cos 30^{\circ}=-1028.717 \mathrm{~L}$,

$\left[\vec{\omega}_{3},\left[\vec{\omega}_{3}, \overrightarrow{P_{3,2} P_{4,3}}\right]\right]_{x}=-\omega_{3}^{2} \overline{D E}=-857.435 \mathrm{~L}$,

$\left[\vec{\omega}_{4},\left[\vec{\omega}_{4}, \overrightarrow{P_{4,3} P_{0,4}}\right]\right]_{x}=0$.

Using equations (40) in (39), angular acceleration of the second member is obtained

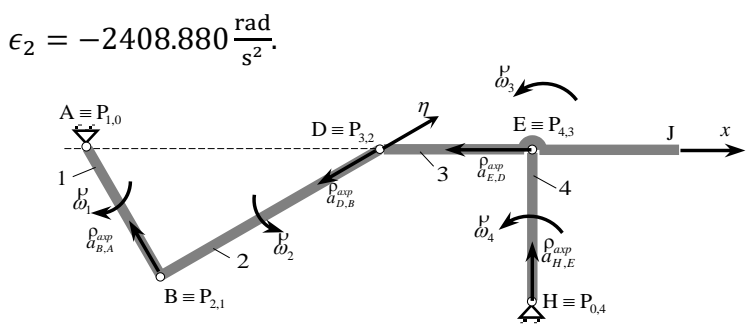

Fig. 8: Example: solution method using co-functions accelerations

By projecting (13) onto $\eta$ axis (Fig. 8), the following is obtained

$M_{\eta}^{\vec{\epsilon}_{1,0}}+M_{\eta}^{\vec{\epsilon}_{2,1}}+M_{\eta}^{\vec{\epsilon}_{3,2}}+M_{\eta}^{\vec{\epsilon}_{4,3}}+M_{\eta}^{\vec{\epsilon}_{0,4}}+\left[\vec{\omega}_{1}\right.$,

$\left.\left[\vec{\omega}_{1}, \overrightarrow{P_{1,0} P_{2,1}}\right]\right]_{\eta}+\left[\vec{\omega}_{2},\left[\vec{\omega}_{2}, \overrightarrow{P_{2,1} P_{3,2}}\right]\right]_{\eta}+\left[\vec{\omega}_{3}\right.$,

$\left.\left[\vec{\omega}_{3}, \overrightarrow{P_{3,2} P_{4,3}}\right]\right]_{\eta}++\left[\vec{\omega}_{4},\left[\vec{\omega}_{4}, \overrightarrow{P_{4,3} P_{0,4}}\right]\right]_{\eta}=0$,

where the terms are:

$M_{\eta}^{\vec{\epsilon}_{2,1}}=0, M_{\eta}^{\vec{\epsilon}_{3,2}}=0, \quad \vec{\epsilon}_{1,0}=\overrightarrow{0} \rightarrow M_{\eta}^{\vec{\epsilon}_{1,0}}=0, \quad \vec{\epsilon}_{0,4}=\overrightarrow{0} \rightarrow$ $M_{\eta}^{\vec{\epsilon}_{0,4}}=0, \quad M_{\eta}^{\vec{\epsilon}_{4,3}}=-\left(\epsilon_{4}-\epsilon_{3}\right) \overline{D E} \sin 30^{0}=\epsilon_{3} L \frac{1}{2^{\prime}}$

$\left[\vec{\omega}_{1},\left[\vec{\omega}_{1}, \overrightarrow{P_{1,0} P_{2,1}}\right]\right]_{\eta}=0,\left[\vec{\omega}_{2},\left[\vec{\omega}_{2}, \overrightarrow{P_{2,1} P_{3,2}}\right]\right]_{\eta}=-\omega_{2}^{2} \overline{B D}=$ $-1187,860 \mathrm{~L}$,

$\left[\vec{\omega}_{3},\left[\vec{\omega}_{3}, \overrightarrow{P_{3,2} P_{4,3}}\right]\right]_{\eta}=-\omega_{3}^{2} \overrightarrow{D E} \cos 30^{\circ}=-742.560 \mathrm{~L}$,

$\left[\vec{\omega}_{4},\left[\vec{\omega}_{4}, \overrightarrow{P_{4,3} P_{0,4}}\right]\right]_{\eta}=\omega_{4}^{2} \overline{E H} \cos 60^{\circ}=800 \mathrm{~L}$

Using equations (41) in (42), angular acceleration of the third member is obtained

$\epsilon_{3}=-2260.840 \frac{\mathrm{rad}}{\mathrm{s}^{2}}$.

\section{Conclusion}

It is shown that kinematic quantities, such as angular velocity and angular acceleration can be obtained using an alternative way, which shows an analogy to static balance equations. Using relative angular velocities and relative angular accelerations, and position vectors of connection points of a mechanism, two vector equations can be established. Using analogy between statics equations and the equations derived here, the first equation, the sum of relative angular velocities equal zero is analog to the sum of forces equal zero in statics. The second equation, the sum of moments of angular velocity vectors with respect to an arbitrary point-pole corresponds to the sum of moments of all forces for an arbitrary point-pole in statics. 
Besides the equations related to $\vec{\omega}$, it is shown here that almost the same analogy can be establish for relative angular acceleration vectors $\vec{\epsilon}$. The equation of relative angular accelerations is identically equal to zero and therefore it does not represent an independent equation. Particularly, it is shown that the sum of moments of angular accelerations along with additional terms that represent the sum of normal relative acceleration denoted as the "axp" components (the components perpendicular to and oriented toward the axis of relative rotation, i.e. toward the connection point) is equal to zero. This equation is analog to the moment equation in statics for an arbitrary point. There is no any restriction for the chosen pole, but it is convenient to choose that point such that the position vector calculation is simplified.

Finally, application of the derived equations was demonstrated through an example of a mechanism for a given instant of motion with given angles at the instant, but the method is valid for an arbitrary instant of a joint-bar mechanism.

\section{References}

Hufnagl B (1974). Mehanizmi I. Univerzitetski Udzbenik, Masinski Fakultet Sarajevo, Sarajevo, Bosnia and Herzegovina.

Ilic B (1966). Mehanizmi II. Naucna knjiga, Beograd, Serbia.

Voloder A (2005). Teorija mehanizama. Masinski Fakultet, Belgrade, Serbia. 